\title{
On a new computational method for the simulation of periodic structures subjected to moving loads
}

\section{Application to vented brake discs}

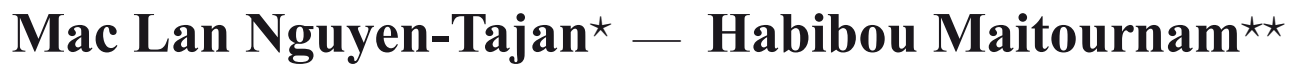 \\ Luis Maestro ${ }^{\star \star \star}$ \\ * PSA Peugeot Citroën, Direction de la Recherche, Route de Gisy \\ F-78943 Vélizy-Villacoublay \\ maclan.nguyen@mpsa.com

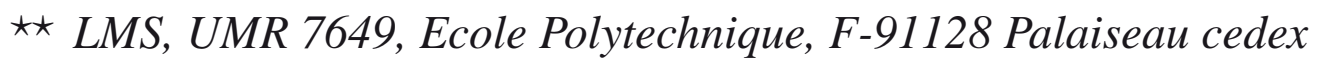 \\ habibou@lms.polytechnique.fr \\ $\star \star \star$ Stagiaire de l'ENSTA
}

\begin{abstract}
The purpose of the paper is to present a new numerical method suitable for the computation of periodic structures subjected to repeated moving loads. It directly derives from the stationary methods proposed for cylindrical and axisymmetrical structures. Its mains features are the use of a calculation reference related to the moving loads and the periodic property of the thermomechanical response. These methods are developped by PSA and the Ecole Polytechnique, in order to design vented brake discs. In this paper, a brief description of the algorithm is first given and examples of numerical simulations of a vented brake disc are treated.
\end{abstract}

RÉSUMÉ. Cet article porte sur le développement d'une nouvelle méthode de résolution numérique adaptée au calcul de structures périodiques soumises à des chargements mobiles et répétés. Il s'inspire directement des méthodes stationnaires développées pour les structures cylindriques ou axisymétriques. Cette méthode repose sur les principes suivants : le repère de calcul est lié au chargement et non plus à la structure, et la réponse thermomécanique de la structure y est supposée périodique. Cette méthode a été développée par PSA Peugeot Citroën et l'Ecole Polytechnique dans le cadre du dimensionnement des disques de frein ventilés. Dans cet article, on donnera une brève description de l'algorithme puis la simulation mécanique d'un disque de frein ventilé illustrera la méthode.

KEYWORDS: periodic steady state algorithm, cyclic moving load, brake disc, thermomechanics. MOTS-CLÉS : algorithme stationnaire périodique, chargement mobile cyclique, disque de frein, thermomécanique. 


\section{Introduction}

The brake is a major security part of a car. In friction braking systems subjected to very severe conditions, various kinds of defects can appear : honeycomb cracking on the rubbing surface, thru-cracks through the disc thickness, fracture of the elbow of the bowl, wear... A numerical model able to predict these phenomena is an alternative and a complement to expensive bench-tests. The computational approach we develop consists in:

- new numerical strategies suitable for problems involving components subjected to moving loads;

- a relevant modelling of the behavior of the material;

- a modelling of the different damage phenomena undergone by the disc, which takes account of the multiaxial and anisothermal characteristics of the loads.

It is essential for the numerical determination of the thermomechanical state of the disc to take into account the main couplings between the different phenomena, the transient characteristic of the thermal history, the inelastic behavior of the material, the non-homogeneous thermomechanical gradients taking place in the disc and the rotation of the disc. The use of classical finite element methods leads to excessive computational times. More precisely, one particularity of the brakes discs is the fact that they are subjected to repeated thermomechanical rotating loads with amplitude varying with the rotations. To simulate a whole braking, dozens of rotations are necessary and dozens of incremental rotations are needed to compute a rotation so that the total computational time is too high. To circumvent this difficulty, algorithms adapted to problems of structures subjected to thermomechanical moving loads have been developed.

The alternative approach consists in using the stationary methods, first proposed by Nguyen and Rahimian [NGU81] and later developed by Dang Van and Maitournam [MAI89, DAN93], which permit to directly calculate the mechanical state of the structure after one rotation or directly the asymptotic state after repeated passes. These algorithms lead to important reductions of the computational time. They can be applied to structures which geometries are generated by the translation or the rotation of a two-dimensional section. Many industrial applications for instance railways [DAN02] and brake discs [NGU02A, NGU02B] have been treated with these methods. In a previous paper [NGU02B], we have treated only solid disc. Here, we consider the case of vented discs (figure 1). These structures have a periodic geometry, so that stationary methods cannot be used in their previous formulations. The objective of the paper is to propose an extension of such methods to periodic structures subjected to moving loads and to quickly determine the mechanical state after a given number of rotations by calculating the solution cycle by cycle. First, we present the principle of the method and its formulation in the case of an elastoplastic material with linear kinematic hardening. Then, an example of simulation of a vented disc is given. 


\section{The periodic stationary method}

\subsection{Overview of the stationary method}

The stationary method was first proposed by Nguyen and Rahimian [NGU81]; Dang Van and Maitournam [MAI89, DAN93] developed it in the case of repeated moving loads. The considered structures are generated by the translation or the rotation of a given 2D section. They are subjected to repeated moving thermomechanical loads. Quasi-static evolution and infinitesimal deformations are assumed. The objective of the pass-by-pass stationary algorithm is to directly determine the thermomechanical response of the structure after each pass of the moving loads.

The stationary method relies on the following hypothesis :

- the amplitude and velocity of the loads remain constant during one load pass;

- in a reference frame attached to the moving loads, the thermomechanical quantities are stationary (steady-state assumption).

The idea is then to use this frame instead of the one related to the structure and so to use eulerian coordinates. The steady state assumption makes the problem become time independent. Internal variables are therefore directly calculated (without time incremental resolutions) by integration along the streamlines which are known as the hypothesis of small transformation holds: it is as if the constitutive law is non local [MAI89, DAN93].

In fact, we consider a continuous medium subjected to a thermomechanical load moving with a velocity $\mathbf{V}(t)$ relatively to a frame $\mathcal{R}=\left(O, \mathbf{e}_{\mathbf{X}}, \mathbf{e}_{\mathbf{Y}}, \mathbf{e}_{\mathbf{Z}}\right)$. We adopt the frame $\mathcal{R}^{\prime}=\left(O^{\prime}, \mathbf{e}_{\mathbf{x}}, \mathbf{e}_{\mathbf{y}}, \mathbf{e}_{\mathbf{z}}\right)$ attached to the load moving. In this frame, the structure is therefore moving with the velocity $-\mathbf{V}(t)$. The material derivative of a tensorial quantity $\mathbf{B}$ related to the material is given by :

$$
\dot{\mathbf{B}}(\mathbf{x}, t)=\frac{\partial \mathbf{B}}{\partial t}(\mathbf{x}, t)+\nabla_{x} \mathbf{B}(\mathbf{x}, t) \cdot \mathbf{v}(\mathbf{x}, t)
$$

with :

$$
\mathbf{v}(\mathbf{x}, t)=\mathbf{v}_{r}(\mathbf{x}, t)-\mathbf{V}(\mathbf{x}, t)
$$

where $\mathbf{x}$ is the geometrical position of the material point in $\mathcal{R}^{\prime}, \mathbf{v}$ the velocity of the material point relatively to $\mathcal{R}^{\prime}$ and $\mathbf{v}_{r}$ its velocity relatively to $\mathcal{R}$.

Thanks to the hypothesis of infinitesinal transformation in the reference linked to the solid, the term $\mathbf{v}_{\mathbf{r}}(\mathbf{x}, t)$ becomes negligible compared to $\mathbf{V}(\mathbf{x}, \mathbf{t})$. Hence, the expression of the material derivative of $\mathbf{B}$ becomes :

$$
\dot{\mathbf{B}}(\mathbf{x}, t)=\frac{\partial \mathbf{B}}{\partial t}(\mathbf{x}, t)-\nabla_{\mathbf{x}} \mathbf{B}(\mathbf{x}, t) \cdot \mathbf{V}(\mathbf{x}, t)
$$


The assumption of steady state in a frame moving with the loads leads to a timeindependent problem for which all time partial derivatives vanish. So the expression of the material derivative of $\mathbf{B}$ becomes :

$$
\dot{\mathbf{B}}(\mathbf{x}, t)=-\nabla_{\mathbf{x}} \mathbf{B}(\mathbf{x}, t) \cdot \mathbf{V}(\mathbf{x}, t)
$$

To numerically solve the problem, we use a frame moving with the loads and replace material derivatives in the governing equations (equilibrium, constitutive law and boundary conditions) by expressions as given above.

\subsection{The periodic stationary method}

We consider a structure with a periodic geometry subjected to a repeated moving load. The load moves with a constant velocity $V \mathbf{e}_{x}$. Its intensity is constant or varies periodically. Although we treat here a rotating load, we choose to present the method for translating load; the two cases are formally identical but the notations are less heavy in the case of translation.

By periodic structure (figure 1), we design a structure which is generated by the translation or the rotation of an inhomogeneous material volume (heterogeneous solid made of different materials or containing voids). An elementary heterogeneous volume is called "cell".

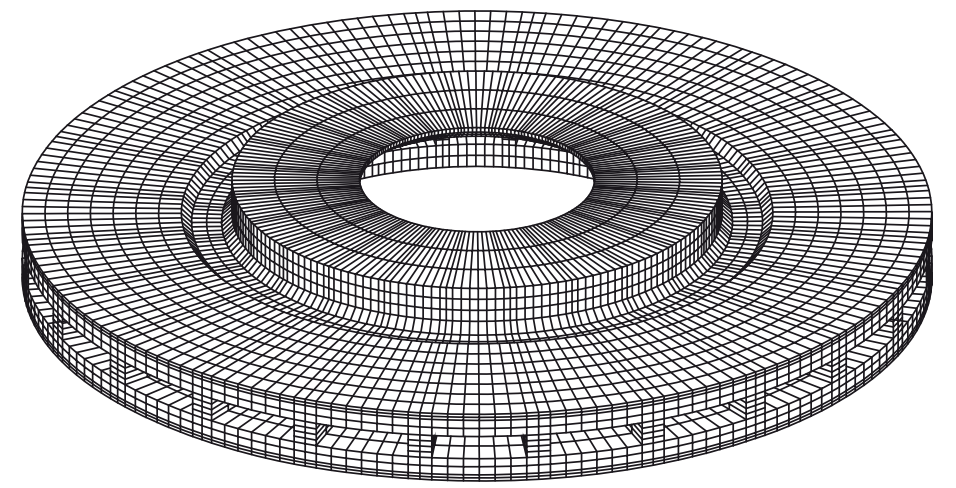

Figure 1. The vented disc: an example of periodic structure

Due to this geometrical and material inhomogeneity, the thermomechanical state of each cell depends on the relative position of the load over the cell. The steady state assumption in the load reference no longer holds. On the other hand, the non stationary behavior is assumed to be periodic. The solution method adopted consists in two main features:

- determination of the transient solution in a time period (time necessary for the loading to move along a cell);

- use of the steady-state assumption at the cells scale in the reference frame related to the moving load. 
So the computations involve two stages: the trial transient elastic solution on a time period will be first sought and then integrations along the streamline associated to the cells are performed (as in the "classical" stationary method).

These two points are used in the formulation of the problem to be numerically solved: on each time interval with a period $T$ ( $T$ is the time necessary for the load to cover the distance $X$ equivalent to the length of a cell along $\mathbf{e}_{x}$ ), the response is variable; it is T-periodic in the load reference.

In other words, in the load reference, for any physical quantity $B$ and for any point $\mathrm{x}$ of the structure:

$$
B(\boldsymbol{x}, t)=B\left(\boldsymbol{x}-X \mathbf{e}_{x}, t-T\right)
$$

This equation allows the transport of the physical quantities from cell to cell along the streamlines, as in the case of the stationary method.

On the other hand, for the transient regime (lasting the time necessary for the load to cover the length of a cell), one has simply $\dot{B}(\mathbf{x}, t)=\frac{\partial B}{\partial t}(\mathbf{x}, t)$ and a method similar to the Large time Increment Method [LAD96] is adopted.

\subsection{Formulation of the periodic stationary method in elastoplasticity}

From the equations (2) and (3), we are able to propose a solution scheme for the periodic stationary method and give the discretized equations of the problem. We just consider here a von Mises elastic-plastic material with a linear kinematic hardening $(c)$ and a yield function $(f)$ of the following form:

$$
f\left(\boldsymbol{\sigma}, c \varepsilon^{p}\right)=\sqrt{\left(\operatorname{dev} \boldsymbol{\sigma}-c \varepsilon^{p}\right):\left(\operatorname{dev} \boldsymbol{\sigma}-c \varepsilon^{p}\right)}-k=\left\|\operatorname{dev} \boldsymbol{\sigma}-c \varepsilon^{p}\right\|-k=\|\boldsymbol{\xi}\|-k
$$

Let us recall that the steady state at the cell scale is assumed; the solution is then entirely determined by the knowledge of the response over a period $(t \in[0, T])$. This time interval is discretized in $m$ instants corresponding to the number of positions of the load on a cell. Practically, we have the following two-stage algorithm.

A global stage consisting in calculating the elastic solution over the whole time interval $[0, T]$ with given internal variables is first carried out: in fact one performs discrete sequence of elastic calculations for all the positions of the load over a particular cell considered as reference.

It is followed by a local stage for the determination of the internal variables by integration along the streamlines, and this is done for all the instants of the interval $[0, T]$. This integration scheme using closest point projection, is detailed in the following.

We denote by $j$ the position of the load on the reference cell, $j \in\{1, \ldots, m\}, \varepsilon_{j}^{p}$ the plastic deformation of the current point of the cell $(n) .(.)_{j}$ denotes quantities at a point of the cell $(n)$, for the $j$ position of the load while $(.)_{j}(n-1)$ denotes quantities at the homologous point of the preceding cell $(n-1)$, for the $j-t h$ position of the 
load. Within, the periodic stationary method, the plastic deformation $\varepsilon^{p}$ is calculated as follows:

If $j \neq 1$ we define $\boldsymbol{\xi}_{j}^{*}=\operatorname{dev} \boldsymbol{\sigma}_{j-1}-c \varepsilon_{j-1}^{p}+2 \mu \Delta(\operatorname{dev} \varepsilon)_{j}$, one has:

- if $\left\|\boldsymbol{\xi}_{j}^{*}\right\|>k$, plastification occurs, so

$$
\begin{aligned}
& \varepsilon_{j}^{p}=\varepsilon_{j-1}^{p}+\frac{1}{2 \mu+C}\left(1-\frac{k}{\left\|\boldsymbol{\xi}_{j}^{*}\right\|}\right) \boldsymbol{\xi}_{j}^{*} \\
& \quad-\text { if }\left\|\boldsymbol{\xi}_{j}^{*}\right\| \leq k, \text { no plastification occurs, so } \\
& \boldsymbol{\varepsilon}_{j}^{p}=\boldsymbol{\varepsilon}_{j-1}^{p}
\end{aligned}
$$

If $j=1$, we define $\boldsymbol{\xi}_{1}^{*}=\operatorname{dev} \boldsymbol{\sigma}_{m-1}(n-1)-c \boldsymbol{\varepsilon}_{m-1}^{p}(n-1)+2 \mu \Delta(\operatorname{dev} \boldsymbol{\varepsilon})_{1}$ one has:

- si $\left\|\boldsymbol{\xi}_{1}^{*}\right\|>k$, plastification occurs, so

$$
\begin{aligned}
& \varepsilon_{1}^{p}=\varepsilon_{m-1}^{p}(n-1)+\frac{1}{2 \mu+C}\left(1-\frac{k}{\left\|\boldsymbol{\xi}_{j}^{*}\right\|}\right) \boldsymbol{\xi}_{j}^{*} \\
& \quad-\text { If }\left\|\boldsymbol{\xi}_{1}^{*}\right\| \leq k, \text { no plastification occurs, so } \\
& \varepsilon_{1}^{p}=\varepsilon_{m-1}^{p}(n-1)
\end{aligned}
$$
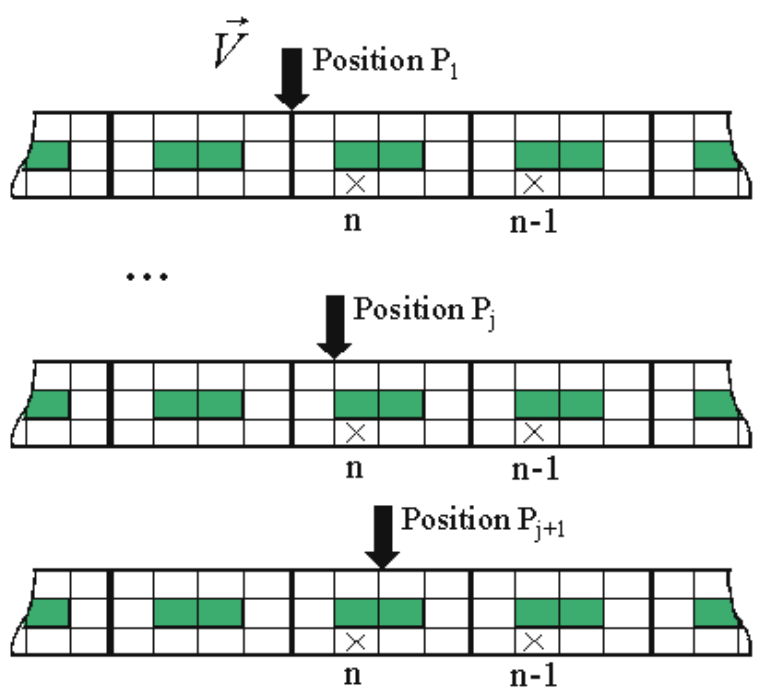

Figure 2. Calculated positions of the load

This algorithm has been implemented in the code Castem 2000 for a von Mises elastic-plastic material with a linear kinematic hardening.

\section{Application to a vented disc}

In this section, we present an application of the periodic stationary method to the vented disc (figure 1). Instead of making a realistic simulation of the braking in which thermal effects are proeminent, we choose to illustrate the different kinds of results that can obtained with this method in the case of purely mechanical problems. 
The dimensions of the disc are the following: external radius $R_{e}=133 \mathrm{~mm}$, internal radius of rubbing surfaces $R_{i}=86.5 \mathrm{~mm}$, thickness of these surfaces $e=13$ $\mathrm{mm}$. The disc constitutive material is cast iron assumed to be, at room temperature, a von Mises elastic-plastic material with a linear kinematic hardening. Its characteristics are: Young modulus $E=110000 \mathrm{MPa}$, Poisson cœfficient $\nu=0.3$, yield limit in traction $\sigma_{y}=90 \mathrm{MPa}$, hardening modulus $h=90000 \mathrm{MPa}$. The figure 3 shows the adopted mesh and the applied loading. The loading consists in two distributions of hertzian pressure (prescribed at the contact zones between the disc and the pads) with the maximum pressure equal to $500 \mathrm{MPa}$. This pressure is greater than the ones encountered during real brakings but here, as thermal effects are not taken into account, we choose a greater pressure just for illustration of the capabilities of the method which is interesting only in inelastic cases. Eight load positions are used for the simulation. They are represented with different colors on figure 3 . Thanks to the periodicity of the solution in the cells "away" from the load, the mesh is truncated; only five cells are represented.

Figure 3. Hertzian pressure distributions on the two surfaces of the vented disc : the eight considered load positions are represented

The evolutions of equivalent plastic deformations during the first pass of the loading are shown on figure 4 for the eight positions of the load. The load is moving in the anti-clock-wise. One can notice that the solution is transient and depends on the relative position of the loading to the cell.

On the first of figures 4, we observe a plastic deformation induced backwards by the moving loads.

On figure 5, one can see the equivalent plastic deformations during the first pass of the loading. The calculation of five successive passes of the loading show that the mechanical stabilized state is reached quickly. The stabilized state is defined by the periodicity of the mechanical response at any material point. On figure 7, we plot the evolution of equivalent plastic deformations along a constant radius streamline: one can then see that the stabilized state is a plastic shakedown. One can also notice 

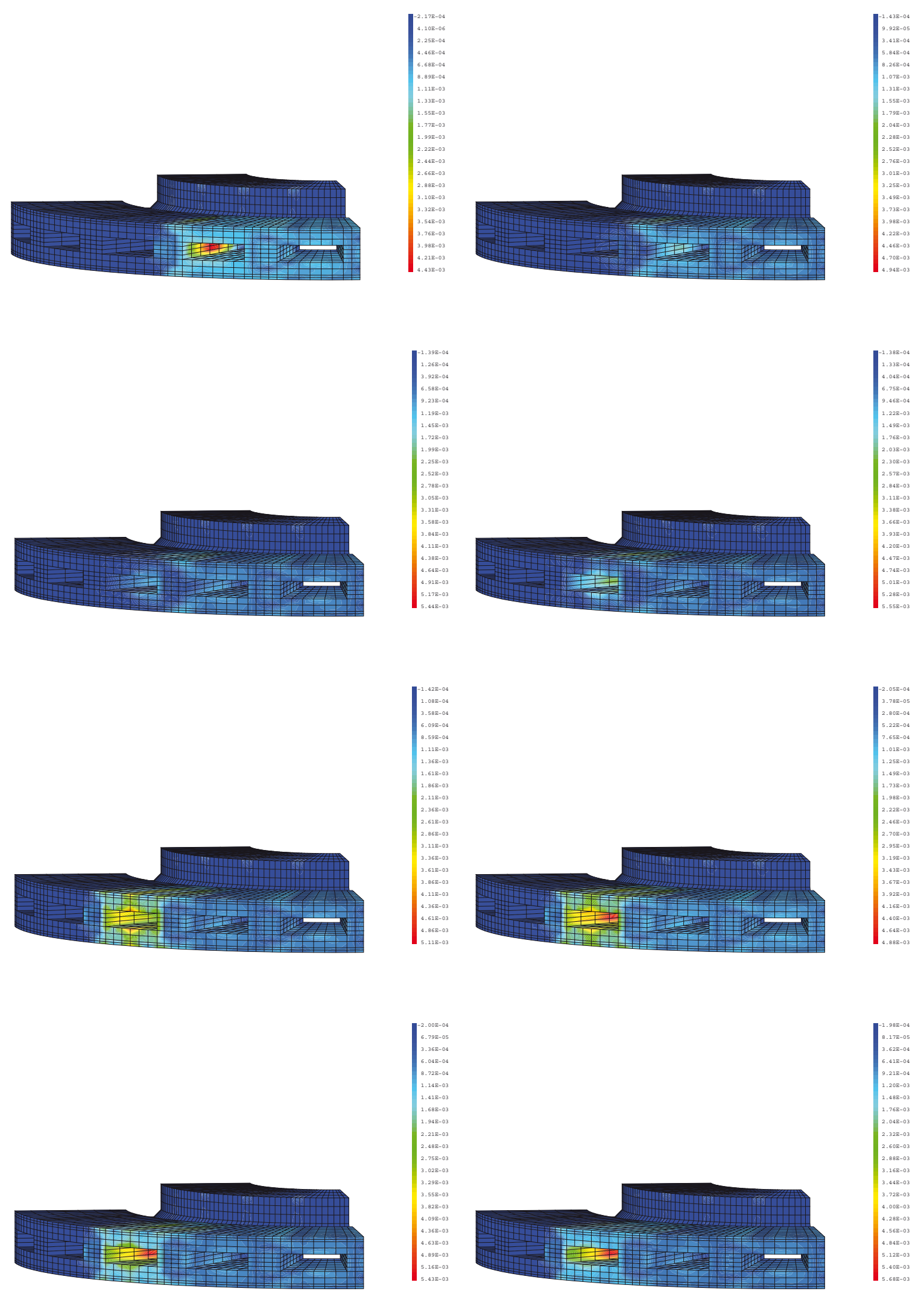

Figure 4. Equivalent plastic deformations during the movement of the load along a cell 


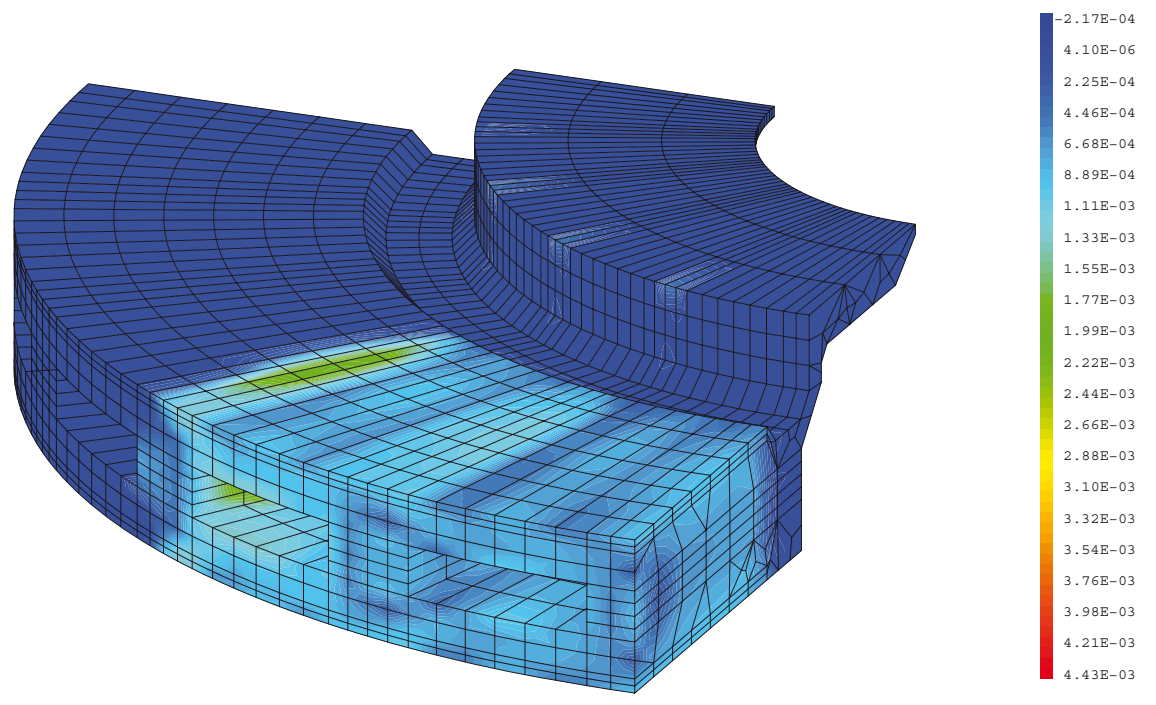

Figure 5. Equivalent plastic deformations after the first pass of the loading
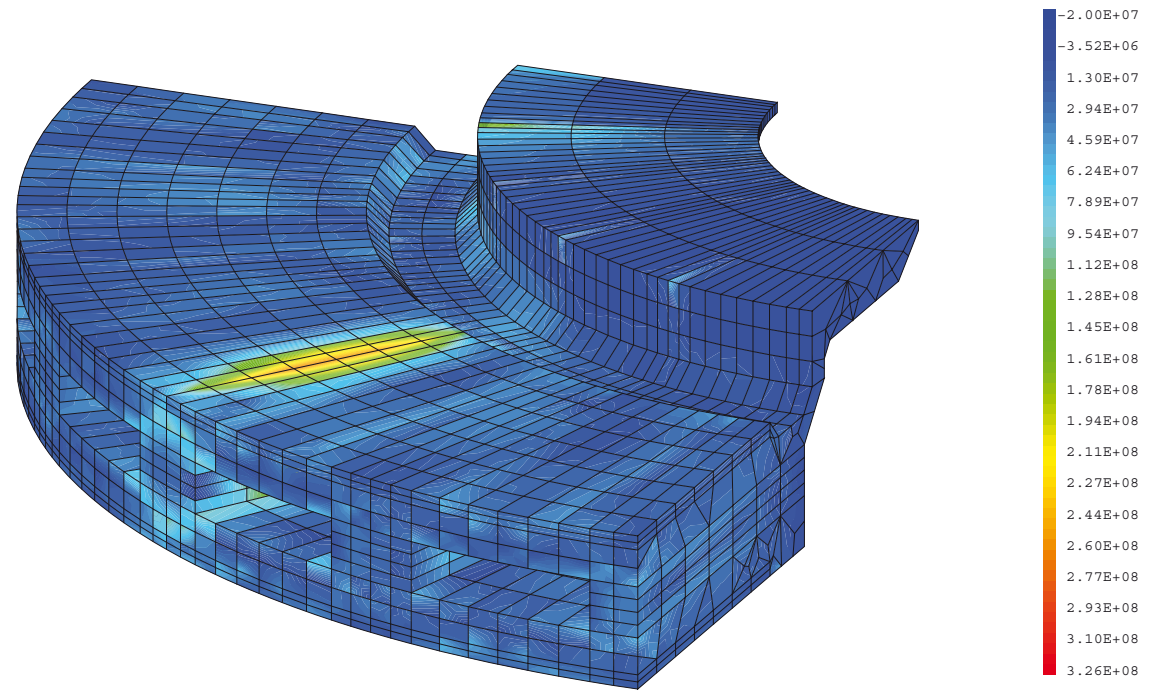

Figure 6. Equivalent stresses at the fifth pass of the loading

that backward, the plastic deformation becomes periodic, with a period equal to the length of a cell $(0.08 \mathrm{~m})$. On figure 6 , we show the equivalent stresses in the limit state obtained for the fifth pass of the loading.

\section{Conclusion}

In this paper, an extension of the stationary methods to periodic inelastic structures subjected to repeated moving loads is presented. It is based on the hypothesis of the periodicity of the response in a reference frame moving with the loads and the use of an approach similar to the Large Time Increment Method. It permits the directly determination of the mechanical state during a whole pass of the loading and therefore the determination of the limit state in the case of repeated moving loads. Even 


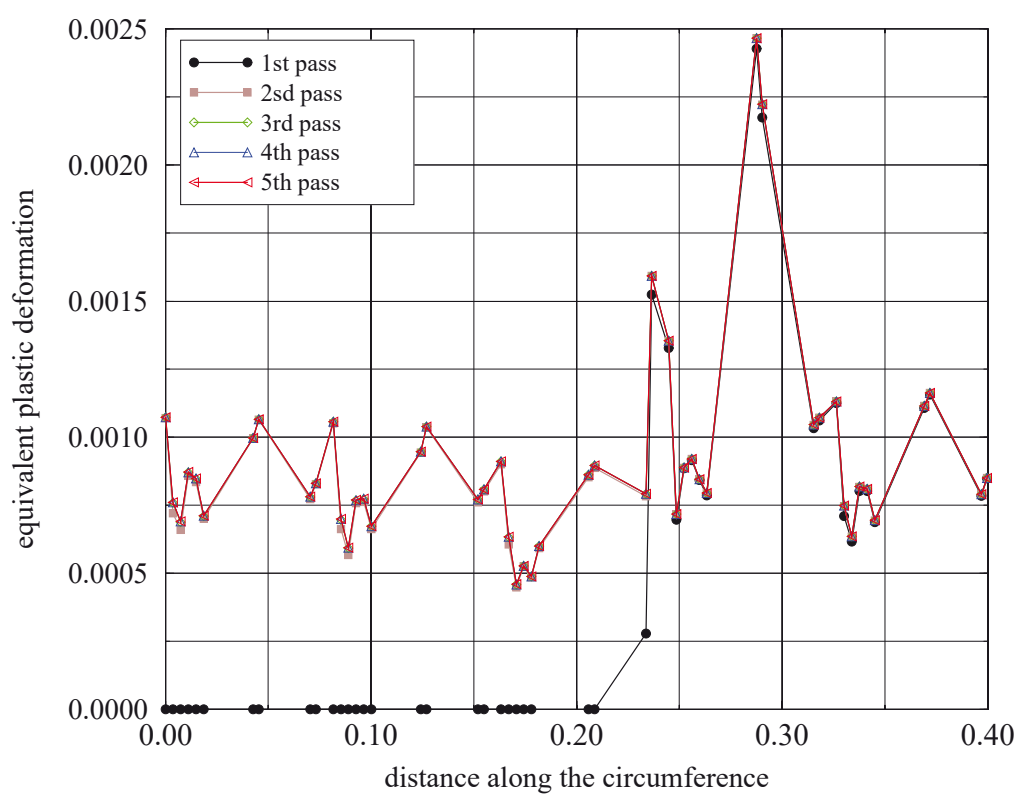

Figure 7. Evolution of equivalent plastic deformations along a streamline for the five first passes of the loading: case of a plastic shakedown

though only the capabilities of this method have be shown, it is clear that it reduces considerably the computational times and accordingly allows the simulation of complicated structures such as vented discs and the determination of asymptotic state of such structures subjected to repeated loads.

\section{Bibliography}

[Dan93] Dang Van K., Maitournam M. H., "Steady-state flow in classical elastoplasticity: Application to repeated rolling and sliding Contact", J. Mech. Phy. Solids, vol. 41, 1993, p. 1691-1710.

[Dan02] Dang Van K., Maitournam M. H., "On some recent trends in modelling of contact fatigue and wear in rail", Wear, vol. 253, 2002, p. 219-227.

[LAD96] LadeVÈze P., Mécanique non linéaires des structures, Hermès, 1996.

[Mai89] Maitournam M. H., "Formulation et résolution numérique des problèmes thermoviscoplastiques en régime permanent", Thèse de doctorat, Ecole Nationale des Ponts et chaussées, 1989.

[Ngu81] NGuYen Q. S., Rahimian M., "Mouvement permanent d'une fissure en milieu élastoplastique", J. de Mech. Appl., vol. 5, 1981, p. 95-120.

[Ngu02a] Nguyen-Tajan T. M. L., "Modélisation thermomécanique des disques de frein par une approche eulérienne", Thèse de doctorat, Ecole Polytechnique, 2002.

[Ngu02B] Nguyen-Tajan T. M. L., Maitournam M. H., Thomas J. J., "Une méthode de calcul de structures soumises à des chargements mobiles. Application au freinage automobile", Revue Européenne des Éléments Finis, vol. 1, 2002, p. 1-15. 